\section{Integration of mental healthcare into primary healthcare in Lagos, Nigeria: the way forward}

\author{
Ayodele O. Coker, ${ }^{1}$ Olufemi B. Olugbile, ${ }^{2}$ \\ Olufemi Oluwatayo ${ }^{3}$ \\ 'Department of Behavioural Medicine, \\ Lagos State University, College of \\ Medicine, Ikeja; ' Lagos State Ministry of \\ Health, Ikeja, Lagos, Nigeria; ${ }^{3}$ Wells Road \\ Centre, Nottinghamshire Healthcare NHS \\ Trust, Nottingham, UK
}

\section{Abstract}

The Lagos State Ministry of Health recently launched its Mental Health Policy aimed at addressing the mental health needs the residents of the State. The policy also aimed at reducing the mental disorders treatment gap in the state by integrating mental healthcare into the primary healthcare in order to make mental healthcare services closer and accessible for residents of the State. This paper therefore critically reviews the rationale for the integration, magnitude of problems in Lagos State with regards to mental healthcare services, available resources, challenges in providing mental healthcare services, recommendations for successful integration, the necessary advocacy needed to implement the integration and benefits of the integration.

\section{Introduction}

Mental and substance use disorders have been severally reported to be common, they tend to become chronic and require long-term commitment to treatment. ${ }^{1,2}$ They were found to constitute the leading cause of disability adjusted life years worldwide accounting for $37 \%$ of healthy life years lost from non-communicable diseases. ${ }^{3,4}$ Previous published reports showed that neuropsychiatric disorders account for $13 \%$ of the global burden of disease and more than $75 \%$ of this burden was found in the low and middle-income countries.,4 Despite many published evidence on the negative impact of mental disorders, only a minority of people with mental disorders receive treatment, and even fewer receive high-quality treatment from mental health experts in the low and middle-income countries. ${ }^{5,6}$ In the light of this, studies showed that between 76 and $84 \%$ of individuals with serious mental illhealth did not receive treatment for their men- tal health disorders representing a very high treatment gap. ${ }^{5,6}$ The World Health Organization (WHO) declared that to reduce the global mental health treatment gap, a possible solution is to integrate mental healthcare services into the primary healthcare (PHC) centers. For this reason, the WHO introduced the Mental Health Gap Action Programme, with the specific aim of scaling up services for mental, substance use and neurological disorders. $^{7-9}$

Lagos State is the formal capital city of Nigeria and it has a population of 18 million people. The State government recently mentioned that the city of Lagos was the fasted growing mega city in the world. ${ }^{10}$ The Lagos State government also claimed that Lagos State was passing through a phase of transformation characterized by rapid population growth and overcrowding with its attendant physical and mental health issues. ${ }^{10}$ The WHOAIMS report on mental health aspects of Nigeria and other published documents from the Lagos State showed that mental healthcare services have been neglected in Nigeria. ${ }^{11-16}$ Nonetheless, the Lagos State government took the initiative of launching its mental health policy. ${ }^{15}$ However, for the policy to be effective, the state must be proactive by looking into the mental health needs of the 18 million residents of the state. This paper therefore discusses the rationale for the integration, magnitude of mental health problems with regards mental healthcare services in the state, available resources, challenges in providing mental healthcare services, recommendations for successful integration, the necessary advocacy needed to implement the integration and the benefits of the integration.

\section{The rationale for integrating mental healthcare services to primary healthcare}

Primary healthcare is the provision of basic essential healthcare made universally accessible to individuals and families in the community as near as possible to where people live and work. ${ }^{7}$ For effective mental healthcare services at the PHC settings, certain issues need to be carried out. The PHC workers are the frontline formal health professionals who are the first level of contact with individuals (and relative families) with physical or mental health disorders within the community. Therefore, medical officers and other medical allied PHC workers need to be trained to acquire the clinical skills of identifying, diagnosing, and managing patients with mental disorders. ${ }^{7}$ When these workers are adequately trained, $\mathrm{PHC}$ will reduce the observed negative
Correspondence: Ayodele 0. Coker, Department of Behavioural Medicine, Lagos State University, College of Medicine, P.M.B. 21266, Ikeja, Nigeria. Tel./Fax: +234.8033267544.

E-mail: cokerrotimi@gmail.com

Key words: Integration; Mental healthcare; Primary healthcare; Lagos, Nigeria.

Received for publication: 15 April 2014. Accepted for publication: 7 November 2014.

This work is licensed under a Creative Commons Attribution 3.0 License (by-nc 3.0).

(O)Copyright A.O. Coker et al., 2015

Licensee PAGEPress, Italy

Healthcare in Low-resource Settings 2015; $3: 3786$ doi:10.4081/hls.2015.3786

implications of those living with severe mental disorders in the Lagos City. The integration will further improve access to mental healthcare within the city, increase acceptability, reduce associated social stigma and human rights abuse, prevent chronicity and physical health comorbidity will likely to be detected early and managed. All specialist and teaching hospitals that offer specialist mental healthcare services are located in downtown Lagos State. However, $80 \%$ of Lagos State dwellers live in rural areas where mental healthcare services are not available. The integration of mental healthcare services in Lagos State will make them available also to those living in rural areas, thus reducing the burden of traveling to the city center to receive treatment in specialist and teaching hospitals which are often associated with labeling and stigmatization.

\section{Available mental healthcare resources}

Lagos State through the Ministry of Health runs a three-tier health system of healthcare: primary (health centers), secondary (general hospitals), and tertiary (teaching) hospital. The State has 51 PHC centers, 21 general hospitals and 1 teaching hospital. Currently, there are no mental healthcare services in any of the primary care centers. There are only seven consultant psychiatrists working for the Lagos State government. Three consultant psychiatrists work at the level of the secondary care in three different general hospitals, while the remaining four consultant psychiatrists work at the State's teaching hospital. Other allied mental healthcare personnel in the State include 30 psychiatric nurses and 2 clinical psychologists. The number of psychiatric 
social workers is quite low and the State cannot boast of any professional occupational therapist. The Lagos State government has only 12 beds at the department of psychiatry of teaching hospital meant solely for academic and didactic purposes. The inadequacy of mental health personnel in Lagos State is another important reason to integrate mental healthcare services into PHC centers. The integration will further bridge the mental illness treatment gap in the State and provide a wider coverage to a large population of Lagosians, especially those living in rural communities. The Lagos State Ministry of Health in 2011 launched its Mental Health Initiative to comprehensively address the State's mental health needs. ${ }^{15}$ Nonetheless, there are few specific mental health programs, such as the one being carried out by a research team from the State's teaching hospital funded by a Canadian grant in which the Mental Health Treatment Gap work book is being used to train PHC workers in just one local government area of the State.

It is however worthy of note that the federal government through the Federal Ministry of Health runs parallel mental healthcare services through the presence of a 476-bed-specialist psychiatric hospital which has 12 consultant psychiatrists; one academic psychiatric department in the University of Lagos Teaching Hospital which has 6 consultant psychiatrists; and a small psychiatric unit in the Military Hospital which has one consultant psychiatrist. The mental healthcare services provided by these federal institutions are meant to augment those being provided by the Lagos State government and are all located at the center of Lagos State. The activities of these federallyfunded hospitals are totally independent from the activities of the Lagos State government and they do not in any way provide mental healthcare services at the primary care centres.

\section{The expected challenges in integrating mental healthcare services into primary health- care services}

The medical personnel at the various $\mathrm{PHC}$ centers in Lagos State include medical officers, registered nurses, community health officers, community health extended workers, and social workers. In order to integrate mental healthcare services to PHC centers, there are likely to be some implementation challenges and they need to be anticipated in order to address them. The expected challenges include: training of the PHC staff who have limited skills in identifying, diagnosing and managing individuals with common mental disorders; reluctance of PHC workers to manage people with mental health disorders because of the cultural and traditional aetiological beliefs of mental illness. Other challenges include inadequate personnel at the PHCs which must be addressed, the probability of increased work load. There must be regular supervision by consultant psychiatrists who must be ready and available to advice and guide the PHC workers on management of patients with mental disorders and who they can refer to in cases of seriously disturbed patients. Likewise, different types of psychotropic medications must always be made available. ${ }^{7,12,1-19}$

\section{Recommendations for a suc- cessful integration}

The Lagos State Ministry of Health has 51 PHC centers, and 21 general hospitals. In order to start the integration, the Ministry should be able to strengthen existing networks of primary and secondary medical services. The integration process must provide adequate funds for recruitment of additional staff and for continuous capacity building of the PHC staff. Generic basic psychotropic medications can be purchased from local pharmaceutical organizations to make them available and affordable for patients. The consultant psychiatrists working at the general hospitals or the State's teaching hospital close to any of the PHC centers should be motivated to closely monitor and supervise the PHC workers and also discuss difficulties encountered in the management of mental disorders at the PHCs. The Lagos State Ministry of Health must also encourage a two-way referral system between the PHCs and secondary and tertiary levels of care. The Ministry must reach out to other non-health sectors for inter-sectorial collaborations. The relevant sectors include the Ministries of Social Welfare, Education, Justice, Prison and Police. Likewise, active collaboration and partnering with religious leaders and non-governmental organisations providing mental healthcare must be encouraged. Psycho-social solutions must also be put in place to assist rehabilitated patients that may require services such as employment, housing and other social services that may alleviate the mental health conditions of patients. There must be regular evaluation and monitoring especially with regards to data collection. Data on patients must be integrated in the general health information system of the State to be used for service improvement.

\section{Advocacy}

In order to provide a successful mental healthcare service to the citizens of Lagos State, the State Government needs to carry out needs-assessment studies in different Local Government Areas of the State. The prevalence of specific mental health and neurological disorders - including alcohol and substance abuse - must be known for effective planning and integration. Thereafter, mental health advocacy and awareness programs should be designed to sensitise the residents of the state on preventive and rehabilitative aspects of mental health disorders. The speciallydesigned advocacy programs should include series of interactive and participatory lectures, symposia, seminars, workshops and outreach mental health programs to bring to awareness signs and symptoms of common mental health disorders at inception, their causes and what needs to be done with regards to help-seeking. In the same vein, the electronic and print media should be involved in the advocacy programs. There should be regular weekly radio and television programs on mental disorders where listeners should be encouraged to phone the mental health specialist on air for further clarifications. The advocacy and awareness programs should also include the making and free distributions of flyers and posters on relevant and concise information on the prevention of mental ill-health. ${ }^{2,7,18}$

\section{The expected benefits of inte- grating mental healthcare serv- ices to primary healthcare services}

The benefits of integrating mental healthcare into primary healthcare have been documented to be enormous. Previously published studies have enumerated the benefits of such integration to include social benefits, understanding and support from nuclear and extended families and from significant members of the community. ${ }^{5,7,8,18-20}$ With regards to cultural benefits, there will be more tolerance for those living with severe and chronic mental disorders. ${ }^{5,18}$ The economic benefits include reduced cost traveling far distance to receive care, and a cost-effective, evidenced-based mental healthcare therapeutic delivery system., ${ }^{5,718}$ The psychological benefits include reduction of social stigma, human rights abuse and negative attitudes towards individual living with psychotic condition in the community which may increase self-esteem of such individuals. $^{20,21}$ The spatial benefits were noted to include proximity of care to patients, and 
increased access to care. The treatment of comorbid physical conditions such as tuberculosis, hypertension and diabetes can also be co-managed which can also lead to better health outcome of patients. ${ }^{5,7,18}$ Thus, prevention and early detection of mental disorders at the primary care level can also reduce chronicity. The integration will prevent individuals with mental illness in the community from being admitted and abused by charlatans, faith-based religious and traditional healers. ${ }^{7,21,22}$ Lastly, capacity building of allied medical professionals at the primary care settings will increase the number of those that can manage mental health disorders within the community. ${ }^{7}$

\section{Conclusions}

The Lagos State government through its Ministry of Health intends to integrate mental healthcare to $\mathrm{PHC}$ centers. The rationale, magnitude of the problems, available resources, and expected challenges benefits were discussed and suggestions preferred. When mental healthcare services are integrated to primary care centers, such services will be taken closer to people in communities within the State. This will encourage those with mental health disorders to seek for help early, and it can lead to reduction of social stigma and human rights violation. It is believed that the effective integration of mental healthcare services into PHCs within the State will eventually reduce the incidence and prevalence of mental health disorders in the State. The advocacy and awareness programs should provide sensitised residents with addresses of various PHCs where mental healthcare services can be received. The PHCs that will provide mental healthcare services must always have adequate and affordable psychotropic medications available at all times. The consultant psychiatrists working for the Lagos State government must be motivated to monitor and attend to difficult psychiatric cases at the PHCs and assist in admitting cases of acutely-disturbed individuals with mental health conditions to their hospitals.

\section{References}

1. WHO. Integrating mental health services into primary healthcare. Geneva, Switzerland: World Health Organization; 2013. Available from: hhp://www.who.int/mental/policy/services/en/index.html

2. Saxena S, Skeen S. No health without mental health: challenges and opportunities in global mental health. Afri J Psychiatry 2012;15:397-400.

3. Whiteford H, Degenhardt L, Rehm J, et al. Global burden of disease attributable to mental and substance use disorders: findings from the Global Burden of Disease Study 2010. Lancet 2013;282:1575-86.

4. Kessler RC, Aguilar-Gaxiola S, Alonso J, et al. The global burden of mental disorders: an update from the World Mental Health (WMH) Surveys. Epidemiol Psychiat S 2009;18:23-33.

5. Lund C, Tomlison M, De Silva M, et al. PRIME: a programme to reduce the treatment gap for mental disorders in five low and middle-income countries. PloS One 2012;9:e1001359.

6. WHO. Mental health systems in low and middle income countries: a cross national analysis of 42 countries using WHO-AIMS data. Geneva, Switzerland: World Health Organization; 2009.

7. WHO. The WHO MIND project: mental improvement for nations development. Geneva, Switzerland: Department of Mental Health and Drug Abuse, World Health Organization; 2008.

8. WHO. Mental Health Gap Action Programme (mhGAP): scaling up care for mental, neurological and substance use disorders. Geneva, Switzerland: World Health Organization; 2007.

9. WHO. Mental Health GAP Action Programme (mhGAP) Newsletter, June 2011. Geneva, Switzerland: World Health Organization; 2011. Available from: http://www.who.int/mental_health/publications/mhgap_newsletters/en/

10. Lagos State Government. Available from: http://www.lsmoh.com/

11. Gureje 0, Saxena S. WHO-AIMS report on mental health system in Nigeria. Ibadan, Nigeria: World Health Organization and
Ministry of Health Publ.; 2006.

12. Olugbile OB, Zachariah MP, Coker AO, et al. Provisions of mental health services in Nigeria. Int Psychiatry 2008;2:27-31.

13. Coker A0, Olugbile OB, Eaton J, Lasebikan VO. Psychiatric psychosocial rehabilitation in Nigeria; what needs to be done. Nigerian J Psychiatry 2011;9:2-9.

14. Lasebikan VO, Ejidokun A, Coker AO. Prevalence of mental disorders and profile of disablement among primary healthcare service users in Lagos Island. Epidemiol Res Intern 2012;2012:357348.

15. Oluwatayo 0, Olugbile 0 , Coker AO. Addressing the mental health needs of a rapidly growing megacity: the new Lagos Mental Health Initiative. Int Psychiatry 2014;11:20-2.

16. Gureje 0, Lasebikan VO. Use of mental health services in a developing country. Results from the Nigerian survey of mental health and well-being. Soc Psych Psych Epid 2006;41:44-9.

17. WHO. Declaration of Alma-Ata. Geneva, Switzerland: World Health Organization; 1978. Available from: www.who.int/publications/almaata_declaration_en.pdf

18. Erinoso L. Community psychiatry in Nigeria: retrospection, challenges, and future prospects. In: Proc. Annual Conf. Association of Psychiatrists in Nigeria, 2010, Enugu, Enugu State, Nigeria.

19. Saraceno B, Vanommeren M, Batniji R, et al. Barriers to improvement of mental health source in low income and middle income countries. Lancet 2007;370:116474.

20. Patel VH, Kirkwood BR, Pednekar S, et al. Improving the outcomes of primary care attenders with common mental disorders in developing countries: a cluster randomized controlled trial of a collaborative stepped care intervention in Goa, India. Trials 2008;9:4.

21. Eaton J, Agomoh A0. Developing mental health services in Nigeria: the impact of a community-based mental health awareness programme. Soc Psych Psych Epid 2008;43:522-8.

22. Adewuya A, Makanjuola R. Preferred treatment for mental illness among Southwestern Nigerians. Psychiat Serv 2009;60: 121-4. 